\title{
Noncommutative Multisoliton Solutions of a Supersymmetric Chiral Model
}

\author{
Xiujuan Zhu \\ Mathematics and Information Technology Institute, Jiangsu Second Normal University, Nanjing 210013, China \\ Correspondence should be addressed to Xiujuan Zhu; yzzhuxiujuan@sina.com
}

Received 6 January 2014; Accepted 27 January 2014; Published 16 March 2014

Academic Editor: Jaume Giné

Copyright ( 2014 Xiujuan Zhu. This is an open access article distributed under the Creative Commons Attribution License, which permits unrestricted use, distribution, and reproduction in any medium, provided the original work is properly cited.

\begin{abstract}
Multisoliton configurations of a superextended and Moyal-type noncommutative deformed modified $2+1$ chiral model have been constructed with the dressing method by Lechtenfeld and Popov several years ago. These configurations have no-scattering property. A two-soliton configuration with nontrivial scattering was constructed soon after that. More multisoliton solutions with general pole data of the superextended and noncommutative Ward model will be constructed in this paper. The method is the supersymmetric and noncommutative extension of Dai and Terng's in constructing soliton solutions of the Ward model.
\end{abstract}

\section{Introduction}

A generalization of the modified $2+1$ chiral model [1] (also called the Ward model in [2]) with $\mathcal{N} \leq 8$ supersymmetries and a Moyal deformation of this model is introduced in [3]. Since the $\mathcal{N}$-extended and deformed chiral model can be formulated as the compatibility conditions of a linear system of differential equations, solutions of this model can be generated with the aid of the system just as in the nonsupersymmetric case $[1,2,4-7]$.

A powerful method, the so-called dressing method, is employed in [3] to construct multisoliton configurations with only simple poles. By allowing for second-order poles in the dressing ansatz, a two-soliton configuration with genuine soliton-soliton interaction is constructed in [8].

In a recent paper [9], we extended the algebraic Bäcklund transformations (BTs) and the order $k$ limiting method of Dai and Terng [2] to the noncommutative case, with which we constructed multisoliton solutions with general pole data of the noncommutative Ward model $[7,10]$. In this paper, we further extend the above case to the supersymmetric one; that is, we use the supersymmetric and noncommutative extended algebraic BTs and the order $k$ limiting method to construct multisoliton solutions with arbitrary poles and multiplicities of the supersymmetric and noncommutative Ward model.

The plan of the paper is as follows: we review the explicit definition of the $\mathcal{N}$-extended and deformed Ward model and the linear system associated to it in Section 2. In Section 3, we first briefly review the dressing construction in [3] and then give the supersymmetric algebraic BTs for the linear system. We apply these algebraic BTs and the superextended order $k$ limiting method to construct a large family of multisoliton configurations with general pole data in Section 4 .

\section{Noncommutative $\mathcal{N}$-Extended Ward Model}

To formulate the noncommutative $\mathcal{N}$-extended Ward model, we need to introduce the following notations $[3,8]$.

(1) $\mathbb{R}^{2,1}=\left(\mathbb{R}^{3}, g\right)$ with coordinates $\left(x^{a}\right)=(x, y, t)$ and the metric $g=\operatorname{diag}(1,1,-1)$.

(2) Grassmann variables (see [11]): some mathematical objects obeying the following anticommutation rules $\chi_{i} \chi_{j}+\chi_{j} \chi_{i}=0$ for any $1 \leq i$, $j \leq n$. In particular, when $i=j$, we have $\chi_{i}^{2}=0$. So any function $K$ of the Grassmann variables is a finite polynomial $K\left(\chi_{1}, \chi_{2}, \ldots, \chi_{n}\right)=$ $\sum_{\alpha_{i}=0,1} a\left(\alpha_{1}, \alpha_{2}, \ldots, \alpha_{n}\right) \chi_{1}^{\alpha_{1}} \chi_{2}^{\alpha_{2}} \cdots \chi_{n}^{\alpha_{n}}$.

(3) The antichiral superspace $\mathbb{R}^{3 / \mathcal{N}}$ with coordinates $\left(x^{a}, \eta_{i}^{\alpha}\right)$ for $\alpha=1,2$ and $i=1, \ldots,(1 / 2) \mathcal{N} \leq 4$; here $\eta_{i}^{\alpha}$ are called fermionic coordinates, which are Grassmann variables. The tensor fields on $\mathbb{R}^{3 \mid \cdot \mathcal{N}}$ are called superfields. 
(4) The noncommutative star product on the antichiral superspace $\mathbb{R}^{3 \mid \mathcal{N}}$ is

$$
\begin{aligned}
& (f \star g)\left(x, y, t, \eta_{i}^{\alpha}\right) \\
& \quad=f\left(x, y, t, \eta_{i}^{\alpha}\right) \exp \left\{\frac{i}{2} \theta\left(\overleftarrow{\partial_{x} \partial_{y}}-\overleftarrow{\partial_{y}} \partial_{x}\right)\right\} g\left(x, y, t, \eta_{i}^{\alpha}\right)
\end{aligned}
$$

Note that the time coordinate remains commutative and no derivatives with respect to the Grassmann variables $\eta_{i}^{\alpha}$.

With the notations listed above, we can now state the definition of this model.

Definition 1 (see $[3,8])$. The $U(n)$-valued superfield $\Phi\left(x^{a}, \eta_{i}^{\alpha}\right)$ of the noncommutative $\mathcal{N}$-extended $U(n)$ Ward model satisfies the classical field equations

$$
\begin{gathered}
\partial_{x}\left(\Phi^{\dagger} \star \partial_{x} \Phi\right)+\partial_{y}\left(\Phi^{\dagger} \star \partial_{y} \Phi\right)-\partial_{t}\left(\Phi^{\dagger} \star \partial_{t} \Phi\right) \\
+\partial_{y}\left(\Phi^{\dagger} \star \partial_{t} \Phi\right)-\partial_{t}\left(\Phi^{\dagger} \star \partial_{y} \Phi\right)=0, \\
\partial_{1}^{i}\left(\Phi^{\dagger} \star \partial_{x} \Phi\right)-\partial_{t}\left(\Phi^{\dagger} \star \partial_{2}^{i} \Phi\right)+\partial_{y}\left(\Phi^{\dagger} \star \partial_{2}^{i} \Phi\right)=0, \\
\partial_{1}^{i}\left(\Phi^{\dagger} \star \partial_{t} \Phi\right)+\partial_{1}^{i}\left(\Phi^{\dagger} \star \partial_{y} \Phi\right)-\partial_{x}\left(\Phi^{\dagger} \star \partial_{2}^{i} \Phi\right)=0, \\
\partial_{1}^{i}\left(\Phi^{\dagger} \star \partial_{2}^{j} \Phi\right)+\partial_{1}^{j}\left(\Phi^{\dagger} \star \partial_{2}^{i} \Phi\right)=0,
\end{gathered}
$$

where $\partial_{\alpha}^{i}:=\partial / \partial \eta_{i}^{\alpha}$, and the unitarity condition

$$
\Phi^{\dagger} \star \Phi=\Phi \star \Phi^{\dagger}=I_{n} .
$$

To avoid cluttering the formulae we suppress the " $\star$ " notation for the supersymmetric version of noncommutative multiplication from now on and most products between classical fields and their components are assumed to be star products until mentioned otherwise.

By [3], model (2) is the compatibility condition for the following linear system of differential equations involving a spectral parameter $\zeta \in \mathbb{C} \cup\{\infty\}$ :

$$
\begin{gathered}
\left(\zeta \partial_{x}-\partial_{u}\right) \psi=\mathscr{A} \psi, \\
\left(\zeta \partial_{v}-\partial_{x}\right) \psi=\mathscr{B} \psi, \\
\left(\zeta \partial_{1}^{i}-\partial_{2}^{i}\right) \psi=\mathscr{C}^{i} \psi, \quad i=1, \ldots, \frac{1}{2} \mathcal{N},
\end{gathered}
$$

where the $n \times n$ matrix $\psi$ depends on $\left(x^{a} \mid \zeta, \eta_{i}^{\alpha}\right)$ and the $n \times n$ matrices $\mathscr{A}, \mathscr{B}$, and $\mathscr{C}^{i}$ are superfields on $\left(x^{a} \mid \eta_{i}^{\alpha}\right) \epsilon$ $\mathbb{R}^{3 \mid \mathcal{N}}$ independent of the spectral parameter $\zeta$. Moreover, $\psi$ is subject to the following reality condition:

$$
\psi\left(x^{a}, \zeta, \eta_{i}^{\alpha}\right)\left[\psi\left(x^{a}, \bar{\zeta}, \eta_{i}^{\alpha}\right)\right]^{\dagger}=I_{n} .
$$

Then the superfield $\Phi\left(x^{a}, \eta_{i}^{\alpha}\right)=\psi\left(x^{a}, 0, \eta_{i}^{\alpha}\right)^{-1}:=\psi^{-1}(\zeta=0)$ clearly satisfies model (2) and unitary condition (3).

The noncommutative star product can be replaced by ordinary product via the Moyal-Weyl map [3, 6, 7]; that is, $f \star g \rightarrow \widehat{f} \widehat{g}$, where $\widehat{f}$ and $\widehat{g}$ are the corresponding operators of $f$ and $g$ under the Moyal-Weyl map, respectively. In later sections, we will mainly use the star-product formulation but use the operator formalism when the former does not work well.

\section{3. $\mathcal{N}$-Extended Multisoliton Configurations with Simple Pole Data}

Dressing method is employed in [3] to construct solutions to the linear system (4). This method is a recursive procedure for generating a new solution from an old one. We briefly review their construction. Rewrite (4) in the form

$$
\begin{gathered}
\psi\left(\partial_{u}-\zeta \partial_{x}\right) \psi^{\dagger}=\mathscr{A}, \\
\psi\left(\partial_{x}-\zeta \partial_{v}\right) \psi^{\dagger}=\mathscr{B}, \\
\psi\left(\partial_{2}^{i}-\zeta \partial_{1}^{i}\right) \psi^{\dagger}=\mathscr{C}^{i}, \quad i=1, \ldots, \frac{1}{2} \mathcal{N},
\end{gathered}
$$

and build a multisoliton solution $\psi_{m}$ with $m$ simple poles at position $\mu_{1}, \ldots, \mu_{m}$ with $\operatorname{Im} \mu_{k}<0$ by left-multiplying an $(m-$ 1) simple pole solution $\psi_{m-1}$ with a single pole factor of the form

$$
I_{n}+\frac{\mu_{m}-\bar{\mu}_{m}}{\zeta-\mu_{m}} P_{m}\left(x^{a}, \eta_{i}^{\alpha}\right),
$$

where the $n \times n$ matrix $P_{m}$ is a Hermitian projection of rank $r_{m}$; that is, $P_{m}^{\dagger}=P_{m}$ and $P_{m}^{2}=P_{m}$; this is obtained by reality condition (5), and therefore one can decompose $P_{m}=$ $T_{m}\left(T_{m}^{\dagger} T_{m}\right)^{-1} T_{m}^{\dagger}$, where $T_{m}$ is an $n \times r_{m}\left(n \geq 2, r_{m} \leq n\right)$ matrix depending on $x^{a}$ and $\eta_{i}^{\alpha}$ and the $r_{m}$ columns of $T_{m}$ span the image of $P_{m}$. Therefore the iteration $\psi_{1} \mapsto \cdots \mapsto \psi_{m}$ yields the multiplicative ansatz

$$
\psi_{m}=\prod_{l=0}^{m-1}\left(I_{n}+\frac{\mu_{m-l}-\bar{\mu}_{m-l}}{\zeta-\mu_{m-l}} P_{m-l}\right),
$$

which, via a partial fraction decomposition, may be written in the additive form

$$
\psi_{m}=I_{n}+\sum_{k=1}^{m} \frac{\Lambda_{m k} S_{k}^{\dagger}}{\zeta-\mu_{k}}
$$

where $\Lambda_{m k}$ and $S_{k}$ are some $n \times r_{k}$ matrices depending on $x^{a}$ and $\eta_{i}^{\alpha}$.

It was shown in [3] that $\psi_{m}$ is the solution of the linear system (6) if

$$
\begin{gathered}
S_{k}=S_{k}\left(w_{k}, \eta_{k}^{i}\right), \\
T_{k}=\left\{\prod_{l=1}^{k-1}\left(I_{n}+\frac{\mu_{k-l}-\bar{\mu}_{k-l}}{\bar{\mu}_{k}-\mu_{k-l}} P_{k-l}\right)\right\} S_{k}
\end{gathered}
$$

with $w_{k}=x+\bar{\mu}_{k} u+\bar{\mu}_{k}^{-1} v$ and $\eta_{k}^{i}=\eta_{i}^{1}+\bar{\mu}_{k} \eta_{i}^{2}$ for $k=1, \ldots, m$. The associated superfield is

$$
\Phi_{m}=\psi_{m}^{-1}(\zeta=0)=\prod_{k=1}^{m}\left(I_{n}-\rho_{k} P_{k}\right) \quad \text { with } \rho_{k}=1-\frac{\mu_{k}}{\bar{\mu}_{k}} .
$$


Thus, the solutions of (2) described by the simple pole ansatz are parametrized by the set $\left\{S_{k}\right\}_{1}^{m}$ of matrix-valued functions of $w_{k}$ and $\eta_{k}^{i}$ and by the pole position $\mu_{k}$.

Example 2. One-soliton configuration

$$
\begin{gathered}
\psi_{1}=I_{n}+\frac{\mu-\bar{\mu}}{\zeta-\mu} P_{1}, \\
\Phi_{1}=\psi_{1}^{-1}(\zeta=0)=I_{n}-\frac{\bar{\mu}-\mu}{\bar{\mu}} P_{1},
\end{gathered}
$$

with $\mu \in \mathbb{C} \backslash \mathbb{R}, P_{1}=T_{1}\left(T_{1}^{\dagger} T_{1}\right)^{-1} T_{1}^{\dagger}$, and $T_{1}=T_{1}\left(w, \eta^{i}\right)$, is an $n \times r$ matrix, where

$$
w=x+\bar{\mu} u+\bar{\mu}^{-1} v, \quad \eta^{i}=\eta_{i}^{1}+\bar{\mu} \eta_{i}^{2}, \quad i=1, \ldots, \frac{1}{2} \mathcal{N} .
$$

This configuration will describe a moving soliton if the matrix $T_{1}$ depends on $w$ rationally $[3,8]$.

We now change the form of the one-soliton solution $\psi_{1}$ in (12) into the following one:

$$
\psi_{1}=I_{n}+\frac{\mu-\bar{\mu}}{\zeta-\mu} P^{\perp},
$$

where $P^{\perp}:=P_{1}$ and thus $P=I_{n}-P^{\perp}=I_{n}-P_{1}=P_{1}^{\perp}$ built from the $n \times r$ matrix $T:=T_{1 \perp}$; then $T_{1}^{\dagger} T=T_{1}^{\dagger} T_{1 \perp}=0$. Since $T_{1}=T_{1}\left(w, \eta^{i}\right)$, we know $T_{1}^{\dagger}=T_{1}^{\dagger}\left(\bar{w}, \bar{\eta}^{i}\right)$; hence $\partial_{w} T_{1}^{\dagger}=0$ and $\partial_{\eta^{i}} T_{1}^{\dagger}=0$. Thus $0=\partial_{w}\left(T_{1}^{\dagger} T\right)=\left(\partial_{w} T_{1}^{\dagger}\right) T+T_{1}^{\dagger}\left(\partial_{w} T\right)=$ $T_{1}^{\dagger}\left(\partial_{w} T\right)$ implies $\partial_{w} T=0$. Similarly, $\partial_{\eta^{i}} T=0$; hence $T=$ $T\left(\bar{w}, \bar{\eta}^{i}\right)=T\left(x+\mu u+\mu^{-1} v, \eta_{i}^{1}+\mu \eta_{i}^{2}\right)$. If we suppose

$$
w=\mu x+\mu^{2} u+v, \quad \eta^{i}=\eta_{i}^{1}+\mu \eta_{i}^{2}, \quad i=1, \ldots, \frac{1}{2} \mathcal{N} .
$$

Then $T=T\left(w, \eta^{i}\right)$ is an $n \times r$ matrix function of $w$ and $\eta^{i}$ and depends on $w$ rationally. With these notations at hand, we can give the following supersymmetric extension of our noncommutative version of algebraic BT, which will be used to construct multisoliton solutions with only simple poles of the linear system (4).

Theorem 3. Let $\psi\left(x^{a}, \zeta, \eta_{i}^{\alpha}\right)$ be a solution of (4) and $\Phi=$ $\psi^{-1}(\zeta=0)$ the associated superfield; choose $\mu \in \mathbb{C} \backslash \mathbb{R}$ such that $\psi$ is holomorphic and nondegenerate at $\zeta=\mu$. Let

$$
\psi_{\mu, P}(\zeta):=I_{n}+\frac{\mu-\bar{\mu}}{\zeta-\mu} P^{\perp}
$$

be a solution of (4) with a simple pole at $\zeta=\mu$, where $P=$ $P\left(x^{a}, \eta_{i}^{\alpha}\right)$ is the Hermitia projection built from an $n \times r$ matrix $T=T\left(w, \eta^{i}\right)$ depending on $w$ rationally. Let $\widetilde{P}=\widetilde{P}\left(x^{a}, \eta_{i}^{\alpha}\right)$ be the Hermitian projection built from the $n \times r$ matrix $\widetilde{T}=$ $\psi\left(x^{a}, \mu, \eta_{i}^{\alpha}\right) T$. Then

(1) $\widetilde{\psi}\left(x^{a}, \zeta, \eta_{i}^{\alpha}\right)=\psi_{\mu, \widetilde{P}}(\zeta) \psi\left(x^{a}, \zeta, \eta_{i}^{\alpha}\right) \psi_{\mu, P}(\zeta)^{-1}$ is holomorphic and nondegenerate at $\zeta=\mu, \bar{\mu}$;
(2) $\psi_{1}=\psi_{\mu, \widetilde{P}} \psi=\widetilde{\psi} \psi_{\mu, P}$ is a new solution to the linear system (4) with some new superfields $\mathscr{A}_{1}, \mathscr{B}_{1}$, and $\mathscr{C}_{1}^{i}$, and the new associated superfield is $\Phi_{1}\left(x^{a}, \eta_{i}^{\alpha}\right)=$ $\Phi\left(\widetilde{P}+(\mu / \bar{\mu}) \widetilde{P}^{\perp}\right)$.

Proof. (1) The residues of the right hand side of $\tilde{\psi}(\zeta)=$ $\psi_{\mu, \widetilde{P}}(\zeta) \psi(\zeta) \psi_{\mu, P}(\zeta)^{-1}$ at $\mu$ and $\bar{\mu}$ are

$$
(\mu-\bar{\mu}) \widetilde{P}^{\perp} \psi(\mu)\left(I_{n}-P^{\perp}\right), \quad(\mu-\bar{\mu})\left(I_{n}-\widetilde{P}^{\perp}\right) \psi(\bar{\mu}) P^{\perp}
$$

respectively, and both equal zero by the definition of $\widetilde{P}$. Thus we have two factorizations of $\psi_{1}=\psi_{\mu, \widetilde{P}} \psi=\widetilde{\psi} \psi_{\mu, P}$.

(2) It suffices to show that $\mathscr{A}_{1}:=\left(\zeta \partial_{x} \psi_{1}-\partial_{u} \psi_{1}\right) \psi_{1}^{-1}$ is independent of the spectral parameter $\zeta$. Using $\psi_{1}=\psi_{\mu, \widetilde{P}} \psi$, we have

$$
\mathscr{A}_{1}=\left(\zeta \partial_{x} \psi_{\mu, \widetilde{P}}-\partial_{u} \psi_{\mu, \widetilde{P}}\right) \psi_{\mu, \widetilde{P}}^{-1}+\psi_{\mu, \widetilde{P}}\left(\zeta \partial_{x} \psi-\partial_{u} \psi\right) \psi^{-1} \psi_{\mu, \widetilde{P}}^{-1}
$$

Since $\left(\zeta \partial_{x} \psi-\partial_{u} \psi\right) \psi^{-1}$ is constant in $\zeta$ by assumption, (18) is holomorphic at $\zeta \in \mathbb{C} \cup\{\infty\} \backslash\{\mu, \bar{\mu}\}$. On the other hand, using $\psi_{1}=\tilde{\psi} \psi_{\mu, P}$, we have

$$
\mathscr{A}_{1}=\left(\zeta \partial_{x} \tilde{\psi}-\partial_{u} \tilde{\psi}\right) \tilde{\psi}^{-1}+\widetilde{\psi}\left(\zeta \partial_{x} \psi_{\mu, P}-\partial_{u} \psi_{\mu, P}\right) \psi_{\mu, P}^{-1} \widetilde{\psi}^{-1} .
$$

Since $\psi_{\mu, P}$ is a solution of $(4),\left(\zeta \partial_{x} \psi_{\mu, P}-\partial_{u} \psi_{\mu, P}\right) \psi_{\mu, P}^{-1}$ is independent of $\zeta$. Together with (1), we deduce that (19) is holomorphic at $\zeta=\mu, \bar{\mu}$. Thus $\mathscr{A}_{1}$ is holomorphic on $\mathbb{C} \cup\{\infty\}$ and hence independent of $\zeta$ by Liouville's theorem. Likewise $\mathscr{B}_{1}:=\left(\zeta \partial_{\nu} \psi_{1}-\partial_{x} \psi_{1}\right) \psi_{1}^{-1}$ and $\mathscr{C}_{1}^{i}:=\left(\zeta \partial_{1}^{i} \psi_{1}-\partial_{2}^{i} \psi_{1}\right) \psi_{1}^{-1}$ are also independent of $\zeta$.

The associated superfield is $\Phi_{1}=\psi_{1}^{-1}(\zeta=0)=\Phi(\widetilde{P}+$ $\left.(\mu / \bar{\mu}) \widetilde{P}^{\perp}\right)$, and then the proof is completed.

Let $\psi_{1}=\psi_{\mu, P} \sharp \psi, \Phi_{1}=\psi_{\mu, P} \sharp \Phi$ denote the $\mathcal{N}$-extended noncommutative algebraic BT generated by $\psi_{\mu, P}$. If we apply these BTs repeatedly (with distinct poles) to a one-soliton solution, then we obtain a multisoliton solution of (4) with simple poles and the associated superfield. Such configurations coincide with the ones constructed in [3], and we call them multisoliton configurations with simple pole data. In contrast, we call solutions of (4) with higher-order poles and the associated fields multisoliton configurations with higherorder pole data, which will be constructed in the next section.

Example 4. Two-soliton configurations with two simple poles.

Let $\mu_{1}, \mu_{2}$ be two distinct complex numbers and $\mu_{1} \neq \bar{\mu}_{2}$, and let $T_{k}\left(w_{k}, \eta_{k}^{i}\right)$ be an $n \times 1$ matrix function depending on $w_{k}$ rationally, where

$$
w_{k}=\mu_{k} x+\mu_{k}^{2} u+v, \quad \eta_{k}^{i}=\eta_{i}^{1}+\mu_{k} \eta_{i}^{2}, \quad i=1, \ldots, \frac{1}{2} \mathcal{N} .
$$

Let $P_{k}=T_{k}\left(T_{k}^{\dagger} T_{k}\right)^{-1} T_{k}^{\dagger}, k=1,2$; then $\psi_{\mu_{1}, P_{1}}$ and $\psi_{\mu_{2}, P_{2}}$ are one-soliton solutions and $\psi_{2}=\psi_{\mu_{2}, P_{2}} \sharp \psi_{\mu_{1}, P_{1}}=\psi_{\mu_{2}, \widetilde{P}_{2}} \psi_{\mu_{1}, P_{1}}$ 
is a two-soliton solution of (4) with two simple poles at $\zeta=$ $\mu_{1}$ and $\zeta=\mu_{2}$, where $\widetilde{P}_{2}$ is the Hermitian projection built from the $n \times 1$ matrix $\widetilde{T}_{2}=\psi_{\mu_{1}, P_{1}}\left(\mu_{2}\right) T_{2}=\left(I_{n}+\left(\left(\mu_{1}-\right.\right.\right.$ $\left.\left.\left.\bar{\mu}_{1}\right) /\left(\mu_{2}-\mu_{1}\right)\right) P_{1}^{\perp}\right) T_{2}$. The associated superfield is $\Phi_{2}=\left(P_{1}+\right.$ $\left.\left(\mu_{1} / \bar{\mu}_{1}\right) P_{1}^{\perp}\right)\left(\widetilde{P}_{2}+\left(\mu_{2} / \bar{\mu}_{2}\right) \widetilde{P}_{2}^{\perp}\right)$.

\section{4. $\mathscr{N}$-Extended Multisoliton Configurations with Higher-Order Pole Data}

A solution of (6) with a double pole at $\zeta=-i$ is constructed in [8] by making a second-order pole at $\zeta=-i$ in the dressing ansatz, that is, considering the following dressing transformation:

$$
\psi=I_{n}-\frac{2 i}{\zeta+i} P \longmapsto \widetilde{\psi}=\left(I_{n}-\frac{2 i}{\zeta+i} \widetilde{P}\right)\left(I_{n}-\frac{2 i}{\zeta+i} P\right),
$$

where the Hermitian projection $P$ is known and $\widetilde{P}=$ $\widetilde{T}\left(\widetilde{T}^{\dagger} \widetilde{T}\right)^{-1} \widetilde{T}^{\dagger}$ is yet to be determined. Demanding that $\widetilde{\psi}$ is again a solution of (6) with some new superfields $\widetilde{\mathscr{A}}, \widetilde{\mathscr{B}}$, and $\widetilde{\mathscr{C}}^{i}$, which are independent of $\zeta$, they obtained the following equations:

$$
\begin{gathered}
\left(I_{n}-\widetilde{P}\right)\left(\partial_{\bar{z}} \widetilde{T}+\left(\partial_{\bar{z}} P\right) \widetilde{T}\right)=0 \\
\left(I_{n}-\widetilde{P}\right)\left(\partial_{t} \widetilde{T}-2 i\left(\partial_{z} P\right) \widetilde{T}\right)=0 \\
\left(I_{n}-\widetilde{P}\right)\left(\frac{1}{2}\left(\partial_{1}^{i}+i \partial_{2}^{i}\right) \widetilde{T}+\left(\partial_{1}^{i} P\right) \widetilde{T}\right)=0
\end{gathered}
$$

After constructing a projection $\widetilde{P}$ via a solution $\widetilde{T}$ of $(22), \widetilde{\psi}$ and the associated superfield $\widetilde{\Phi}=\widetilde{\psi}^{-1}(\zeta=0)=\left(I_{n}-2 P\right)\left(I_{n}-\right.$ $2 \widetilde{P})$ are derived.

Their construction of the solution $\widetilde{T}$ is inspired by the known form of $\widetilde{T}$ in the bosonic case $[4,5]$, that is, making the ansatz $\widetilde{T}=T+T_{\perp}\left(T_{\perp}^{\dagger} T_{\perp}\right)^{-1} g$ with $T_{\perp}$ orthogonal to $T$.

Below we will present a supersymmetric extension of our noncommutative limiting method as in the bosonic case to construct multisoliton configurations with a higherorder pole at $\zeta=\mu$. We give an example of two-soliton configuration with a second-order pole by taking a limit at first.

Example 5. Two-soliton configuration with a second-order pole.

Let $\mu \in \mathbb{C} \backslash \mathbb{R}, f, g$ be two holomorphic functions of $\left(w, \eta^{i}\right)$ and depend on the bosonic variable $w$ rationally:

$$
T_{1}=\left(\begin{array}{c}
1 \\
f\left(w, \eta^{i}\right)
\end{array}\right), \quad T_{2}=\left(\begin{array}{c}
1 \\
f\left(w_{\epsilon}, \eta_{\epsilon}^{i}\right)+\epsilon g\left(w_{\epsilon}, \eta_{\epsilon}^{i}\right)
\end{array}\right)
$$

where $w=\mu x+\mu^{2} u+v$ and $\eta^{i}=\eta_{i}^{1}+\mu \eta_{i}^{2}, w_{\epsilon}=(\mu+\epsilon) x+(\mu+$ $\epsilon)^{2} u+v$ and $\eta_{\epsilon}^{i}=\eta_{i}^{1}+(\mu+\epsilon) \eta_{i}^{2}, i=1, \ldots,(1 / 2) \mathcal{N}$, for any $\epsilon \in \mathbb{C}$ with $|\epsilon|$ small, $P_{1}=T_{1}\left(T_{1}^{\dagger} T_{1}\right)^{-1} T_{1}^{\dagger}$, and $P_{2}=T_{2}\left(T_{2}^{\dagger} T_{2}\right)^{-1} T_{2}^{\dagger}$. The Taylor expansion of $f\left(w_{\epsilon}, \eta_{\epsilon}^{i}\right)$ in $\epsilon$ is

$$
\begin{aligned}
f\left(w_{\epsilon}, \eta_{\epsilon}^{i}\right)= & f\left(w, \eta^{i}\right)+\frac{\partial f}{\partial w}\left(w_{\epsilon}-w\right) \\
& +\sum_{i=1}^{(1 / 2) \mathcal{N}} \frac{\partial f}{\partial \eta^{i}}\left(\eta_{\epsilon}^{i}-\eta^{i}\right)+O\left(\epsilon^{2}\right) \\
= & f\left(w, \eta^{i}\right)+\left[\frac{\partial f}{\partial w}(x+2 \mu u)+\sum_{i=1}^{(1 / 2) \mathcal{N}} \frac{\partial f}{\partial \eta^{i}} \eta_{i}^{2}\right] \epsilon \\
& +O\left(\epsilon^{2}\right),
\end{aligned}
$$

where $\partial f / \partial \eta^{i}$ is right derivative of $f$ with respect to the Grassmann variable $\eta^{i}$. Note that the products in the expansion are the ordinary products and that we omit the variables $\left(w, \eta^{i}\right)$ of $\partial f / \partial w$ and $\partial f / \partial \eta^{i}$ for simplicity. Clearly, $g\left(w_{\epsilon}, \eta_{\epsilon}^{i}\right)$ has similar expansion; therefore

$$
\begin{aligned}
f\left(w_{\epsilon}, \eta_{\epsilon}^{i}\right)+\epsilon g\left(w_{\epsilon}, \eta_{\epsilon}^{i}\right) \\
=f\left(w, \eta^{i}\right)+\left[\frac{\partial f}{\partial w}(x+2 \mu u)\right. \\
\\
\left.+\sum_{i=1}^{(1 / 2) \mathcal{N}} \frac{\partial f}{\partial \eta^{i}} \eta_{i}^{2}+g\left(w, \eta^{i}\right)\right] \epsilon+O\left(\epsilon^{2}\right) .
\end{aligned}
$$

Then

$$
\begin{aligned}
& \widetilde{T}_{2}=\psi_{\mu_{1}, P_{1}}(\mu+\epsilon) T_{2} \\
& =\left(I_{n}+\frac{\mu_{1}-\bar{\mu}_{1}}{\epsilon} P_{1}^{\perp}\right) \\
& \times\left(f+\left[\frac{\partial f}{\partial w}(x+2 \mu u)+\sum_{i=1}^{1} \frac{\partial f}{\partial \eta^{i}} \eta_{i}^{2}+g\right] \epsilon+O\left(\epsilon^{2}\right)\right) \\
& =\left(\begin{array}{l}
1 \\
f
\end{array}\right)+(\mu-\bar{\mu}) P_{1}^{\perp} \\
& \times\left(\frac{\partial f}{\partial w}(x+2 \mu u)+\sum_{i=1}^{0} \frac{\partial f}{\partial \eta^{i}} \eta_{i}^{2}+g\right)+O(\epsilon) ; \\
& \widehat{T}_{2}=\lim _{\epsilon \rightarrow 0} \widetilde{T}_{2}=\left(\begin{array}{l}
1 \\
f
\end{array}\right)+(\mu-\bar{\mu}) P_{1}^{\perp} \\
& \times\left(\frac{\partial f}{\partial w}(x+2 \mu u)+\sum_{i=1}^{0} \frac{\partial f}{\partial \eta^{i}} \eta_{i}^{2}+g\right) .
\end{aligned}
$$


Let $\widetilde{P}_{2}=\widetilde{T}_{2}\left(\widetilde{T}_{2}^{\dagger} \widetilde{T}_{2}\right)^{-1} \widetilde{T}_{2}^{\dagger}$ and $\widehat{P}_{2}=\widehat{T}_{2}\left(\widehat{T}_{2}^{\dagger} \widehat{T}_{2}\right)^{-1} \widehat{T}_{2}^{\dagger}$, then

$$
\begin{aligned}
\widehat{\psi}_{2}=\psi_{\mu, \widehat{P}_{2}} \psi_{\mu, P_{1}} & =\lim _{\epsilon \rightarrow 0} \psi_{\mu+\epsilon, \widetilde{P}_{2}} \psi_{\mu, P_{1}} \\
& =\lim _{\epsilon \rightarrow 0} \psi_{\mu+\epsilon, P_{2}} \sharp \psi_{\mu, P_{1}}
\end{aligned}
$$

is a two-soliton solution of (4) with a double pole at $\zeta=\mu$, and the associated superfield is

$$
\widehat{\Phi}_{2}=\widehat{\psi}_{2}^{-1}(\zeta=0)=\left(P_{1}+\frac{\mu}{\bar{\mu}} P_{1}^{\perp}\right)\left(\widehat{P}_{2}+\frac{\mu}{\bar{\mu}} \widehat{P}_{2}^{\perp}\right) .
$$

Next, we use a systematic limiting method and our extended algebraic BTs in Section 3 to construct multisoliton solutions of (4) with pole data $(\mu, k)$ for any $\mu \in \mathbb{C} \backslash \mathbb{R}$ and $k \geq 2$.

Let $\mu \in \mathbb{C} \backslash \mathbb{R}$ be a constant and $\left\{a_{j}\left(w, \eta^{i}\right)\right\}_{j=0}^{\infty}$ a sequence of $n \times 1$ matrix functions depending on $w$ rationally. For any $\epsilon \epsilon$ $\mathbb{C}$ with $|\epsilon|$ small, let $w=\mu x+\mu^{2} u+v, \eta^{i}=\eta_{i}^{1}+\mu \eta_{i}^{2}, w_{\epsilon}=(\mu+$ $\epsilon) x+(\mu+\epsilon)^{2} u+v$, and $\eta_{\epsilon}^{i}=\eta_{i}^{1}+(\mu+\epsilon) \eta_{i}^{2}$, for $i=1, \ldots,(1 / 2) \mathcal{N}$. Here we only consider the case of $\mathcal{N}=8$ since it is easily truncated to any smaller even number $\mathcal{N}$ of supersymmetries (see [8]); thus

$$
\begin{aligned}
& w_{\epsilon}-w=(x+2 \mu u) \epsilon+u \epsilon^{2}, \\
& \eta_{\epsilon}^{i}-\eta^{i}=\eta_{i}^{2} \epsilon, \quad i=1, \ldots, 4 .
\end{aligned}
$$

Let $F_{k, \epsilon}=\sum_{j=0}^{k} a_{j}\left(w_{\epsilon}, \eta_{\epsilon}^{i}\right) \epsilon^{j}$ for $k \geq 1$. The Taylor expansion of $a_{j}\left(w_{\epsilon}, \eta_{\epsilon}^{i}\right)$ at $\left(w, \eta^{i}\right)$ is

$$
\begin{aligned}
& a_{j}\left(w_{\epsilon}, \eta_{\epsilon}^{i}\right) \\
&=a_{j}\left(w, \eta^{i}\right)+\frac{\partial a_{j}}{\partial w}\left(w_{\epsilon}-w\right)+\sum_{i=1}^{4} \frac{\partial a_{j}}{\partial \eta^{i}}\left(\eta_{\epsilon}^{i}-\eta^{i}\right) \\
&+\frac{1}{2 !}\left[\frac{\partial^{2} a_{j}}{\partial w^{2}}\left(w_{\epsilon}-w\right)^{2}\right. \\
&+2 \sum_{i=1}^{4} \frac{\partial^{2} a_{j}}{\partial w \partial \eta^{i}}\left(w_{\epsilon}-w\right)\left(\eta_{\epsilon}^{i}-\eta^{i}\right) \\
&\left.+\sum_{i, k=1}^{4} \frac{\partial^{2} a_{j}}{\partial \eta^{i} \partial \eta^{k}}\left(\eta_{\epsilon}^{k}-\eta^{k}\right)\left(\eta_{\epsilon}^{i}-\eta^{i}\right)\right] \\
&+\frac{\partial^{3} a_{j}}{3 !}\left(w_{\epsilon}-w\right)^{3} \\
&+3 \sum_{i=1}^{4} \frac{\partial^{3} a_{j}}{\partial w^{2} \partial \eta^{i}}\left(w_{\epsilon}-w\right)^{2}\left(\eta_{\epsilon}^{i}-\eta^{i}\right) \\
&+3 \sum_{i, k=1}^{4} \frac{\partial^{3} a_{j}}{\partial w \partial \eta^{i} \partial \eta^{k}} \\
&+\left(w_{\epsilon}-w\right)\left(\eta_{\epsilon}^{k}-\eta^{k}\right)\left(\eta_{\epsilon}^{i}-\eta^{i}\right)
\end{aligned}
$$

$$
\begin{aligned}
+\sum_{i, k, l=1}^{4} \frac{\partial^{3} a_{j}}{\partial \eta^{i} \partial \eta^{k} \partial \eta^{l}}\left(\eta_{\epsilon}^{l}-\eta^{l}\right) \\
\left.\quad \times\left(\eta_{\epsilon}^{k}-\eta^{k}\right)\left(\eta_{\epsilon}^{i}-\eta^{i}\right)\right]
\end{aligned}
$$$$
+\frac{1}{4 !}\left[\frac{\partial^{4} a_{j}}{\partial w^{4}}\left(w_{\epsilon}-w\right)^{4}\right.
$$$$
+4 \sum_{i=1}^{4} \frac{\partial^{4} a_{j}}{\partial w^{3} \partial \eta^{i}}\left(w_{\epsilon}-w\right)^{3}\left(\eta_{\epsilon}^{i}-\eta^{i}\right)
$$$$
+6 \sum_{i, k=1}^{4} \frac{\partial^{4} a_{j}}{\partial w^{2} \partial \eta^{i} \partial \eta^{k}}\left(w_{\epsilon}-w\right)^{2}
$$$$
\times\left(\eta_{\epsilon}^{k}-\eta^{k}\right)\left(\eta_{\epsilon}^{i}-\eta^{i}\right)
$$$$
+4 \sum_{i, k, l=1}^{4} \frac{\partial^{4} a_{j}}{\partial w \partial \eta^{i} \partial \eta^{k} \partial \eta^{l}}\left(w_{\epsilon}-w\right)
$$$$
\times\left(\eta_{\epsilon}^{l}-\eta^{l}\right)\left(\eta_{\epsilon}^{k}-\eta^{k}\right)\left(\eta_{\epsilon}^{i}-\eta^{i}\right)
$$$$
+\sum_{i, k, l, m=1}^{4} \frac{\partial^{4} a_{j}}{\partial \eta^{i} \partial \eta^{k} \partial \eta^{l} \partial \eta^{m}}
$$$$
\times\left(\eta_{\epsilon}^{m}-\eta^{m}\right)\left(\eta_{\epsilon}^{l}-\eta^{l}\right)
$$$$
\left.\times\left(\eta_{\epsilon}^{k}-\eta^{k}\right)\left(\eta_{\epsilon}^{i}-\eta^{i}\right)\right]+\cdots,
$$

where the sums are taken over different indices when there are more than one index and the partial derivatives of $a_{j}\left(w, \eta^{i}\right)$ with respect to the Grassmann variables are given by the following remark.

Remark 6. We can write

$$
\begin{aligned}
a_{j}\left(w, \eta^{i}\right)= & a_{j, 0}(w)+\sum_{i=1}^{4} a_{j, i}(w) \eta^{i} \\
& +\sum_{k, i=1}^{4} a_{j, k i}(w) \eta^{k} \eta^{i} \\
& +\sum_{l, k, i=1}^{4} a_{j, l k i}(w) \eta^{l} \eta^{k} \eta^{i} \\
& +\sum_{m, l, k, i=1}^{4} a_{j, m l k i}(w) \eta^{m} \eta^{l} \eta^{k} \eta^{i}
\end{aligned}
$$

hence the right derivatives of $a_{j}\left(w, \eta^{i}\right)$ with respect to one or several of the Grassmann variables are computed as follows:

$$
\begin{aligned}
\frac{\partial a_{j}}{\partial \eta^{i}}= & a_{j, i}+\sum_{\substack{k=1 \\
k \neq i}}^{4} a_{j, k i} \eta^{k}+\sum_{\substack{l, k=1 \\
l, k \neq i}}^{4} a_{j, l k i} \eta^{l} \eta^{k} \\
& +\sum_{\substack{m, l, k=1 \\
m, l, k \neq i}}^{4} a_{j, m l k i} \eta^{m} \eta^{l} \eta^{k},
\end{aligned}
$$




$$
\begin{gathered}
\frac{\partial^{2} a_{j}}{\partial \eta^{i} \partial \eta^{k}}=a_{j, k i}-a_{j, i k}+\sum_{\substack{l=1 \\
l \neq i, k}}^{4}\left(a_{j, l k i}-a_{j, l i k}\right) \eta^{l} \\
+\sum_{\substack{m, l=1 \\
m, l \neq i, k}}^{4}\left(a_{j, m l k i}-a_{j, m l i k}\right) \eta^{m} \eta^{l}, \\
\frac{\partial^{3} a_{j}}{\partial \eta^{i} \partial \eta^{k} \partial \eta^{l}}=\sum_{\sigma} \operatorname{sign} \sigma a_{j, \sigma(l) \sigma(k) \sigma(i)} \\
+\sum_{\substack{m=1 \\
m \neq i, k, l}}^{4} \sum_{\sigma} \operatorname{sign} \sigma a_{j, m \sigma(l) \sigma(k) \sigma(i)} \eta^{m}, \\
\frac{\partial^{4} a_{j}}{\partial \eta^{i} \partial \eta^{k} \partial \eta^{l} \partial \eta^{m}}=\sum_{\tau} \operatorname{sign} \tau a_{j, \tau(m) \tau(l) \tau(k) \tau(i)},
\end{gathered}
$$

where $\sum_{\sigma}, \sum_{\tau}$ represent that the sums are taken over the permutation group of $\{l, k, i\}$ and $\{m, l, k, i\}$, respectively.

Substituting (29) into the Taylor expansion of $a_{j}\left(w_{\epsilon}, \eta_{\epsilon}^{i}\right)$, we get the Taylor expansion of $a_{j}\left(w_{\epsilon}, \eta_{\epsilon}^{i}\right)$ in $\epsilon$. Assume that $a_{j}\left(w_{\epsilon}, \eta_{\epsilon}^{i}\right)=\sum_{p=0}^{\infty} b_{j, p} \epsilon^{p}$, then

$$
\begin{aligned}
b_{j, 0}= & a_{j}\left(w, \eta^{i}\right), \\
b_{j, 1}= & \frac{\partial a_{j}}{\partial w}(x+2 \mu u)+\sum_{i=1}^{4} \frac{\partial a_{j}}{\partial \eta^{i}} \eta_{i}^{2}, \\
b_{j, 2}= & \frac{\partial a_{j}}{\partial w} u+\frac{1}{2} \frac{\partial^{2} a_{j}}{\partial w^{2}}(x+2 \mu u)^{2} \\
& +\sum_{i=1}^{4} \frac{\partial^{2} a_{j}}{\partial w \partial \eta^{i}}(x+2 \mu u) \eta_{i}^{2} \\
& +\frac{1}{2} \sum_{i, k=1}^{4} \frac{\partial^{2} a_{j}}{\partial \eta^{i} \partial \eta^{k}} \eta_{k}^{2} \eta_{i}^{2}, \\
& \partial^{2} a_{j} \\
b_{j, 3} & \frac{\partial w^{2}}{x+2 \mu u) u} \\
& +\sum_{i=1}^{4} \frac{\partial^{2} a_{j}}{\partial w \partial \eta^{i}} u \eta_{i}^{2}+\frac{1}{6} \frac{\partial^{3} a_{j}}{\partial w^{3}}(x+2 \mu u)^{3} \\
& +\frac{1}{6} \sum_{i=1}^{4} \frac{\partial^{3} a_{j}}{\partial w^{2} \partial \eta^{i}}(x+2 \mu u)^{2} \eta_{i}^{2} \\
& +\frac{1}{2} \sum_{i, k=1}^{4} \frac{\partial^{3} a_{j}}{\partial w \partial \eta^{i} \partial \eta^{k}}(x+2 \mu u) \eta_{k}^{2} \eta_{i}^{2} \\
&
\end{aligned}
$$

$$
\begin{aligned}
& b_{j, 4}= \frac{1}{2} \frac{\partial^{2} a_{j}}{\partial w^{2}} u^{2}+\frac{1}{2} \frac{\partial^{3} a_{j}}{\partial w^{3}}(x+2 \mu u)^{2} u \\
&+\sum_{i=1}^{4} \frac{\partial^{3} a_{j}}{\partial w^{2} \partial \eta^{i}}(x+2 \mu u)^{2} u \eta_{i}^{2} \\
&+\frac{1}{2} \sum_{i, k=1}^{4} \frac{\partial^{3} a_{j}}{\partial w \partial \eta^{i} \partial \eta^{k}} u \eta_{k}^{2} \eta_{i}^{2} \\
&+\frac{1}{24} \frac{\partial^{4} a_{j}}{\partial w^{4}}(x+2 \mu u)^{4} \\
&+\frac{1}{6} \sum_{i=1}^{4} \frac{\partial^{4} a_{j}}{\partial w^{3} \partial \eta^{i}}(x+2 \mu u)^{3} \eta_{i}^{2} \\
&+\frac{1}{4} \sum_{i, k=1}^{4} \frac{\partial^{4} a_{j}}{\partial w^{2} \partial \eta^{i} \partial \eta^{k}}(x+2 \mu u)^{2} \eta_{k}^{2} \eta_{i}^{2} \\
&+\frac{1}{6} \sum_{i, k, l=1}^{4} \frac{\partial^{4} a_{j}}{\partial w \partial \eta^{i} \partial \eta^{k} \partial \eta^{l}} \\
&+\frac{1}{24} \sum_{i, k, l, m=1}^{4} \frac{\partial^{4} a_{j}}{\partial \eta^{i} \partial \eta^{k} \partial \eta^{l} \partial \eta^{m}} \\
& \times(x+2 \mu u) \eta_{l}^{2} \eta_{k}^{2} \eta_{i}^{2} \\
& 2 \\
& 2
\end{aligned}
$$

Expanding $F_{k, \epsilon}$ in $\epsilon$, we obtain

$$
\begin{aligned}
F_{k, \epsilon} & =\sum_{j=0}^{k} a_{j}\left(w_{\epsilon}, \eta_{\epsilon}^{i}\right) \epsilon^{j} \\
& =\sum_{j=0}^{k} \sum_{p=0}^{\infty} b_{j, p} \epsilon^{j+p} \\
& =c_{0}+c_{1} \epsilon+\cdots+c_{k} \epsilon^{k}+O\left(\epsilon^{k+1}\right),
\end{aligned}
$$

where $c_{p}=\sum_{j=0}^{p} b_{j, p-j}$ are given as follows:

$$
\begin{aligned}
c_{0}= & a_{0}\left(w, \eta^{i}\right) \\
c_{1}= & \frac{\partial a_{0}}{\partial w}(x+2 \mu u)+\sum_{i=1}^{4} \frac{\partial a_{0}}{\partial \eta^{i}} \eta_{i}^{2}+a_{1}\left(w, \eta^{i}\right) \\
c_{2}= & \frac{\partial a_{0}}{\partial w} u+\frac{1}{2} \frac{\partial^{2} a_{0}}{\partial w^{2}}(x+2 \mu u)^{2} \\
& +\sum_{i=1}^{4} \frac{\partial^{2} a_{0}}{\partial w \partial \eta^{i}}(x+2 \mu u) \eta_{i}^{2}
\end{aligned}
$$




$$
\begin{aligned}
& +\frac{1}{2} \sum_{i, k=1}^{4} \frac{\partial^{2} a_{0}}{\partial \eta^{i} \partial \eta^{k}} \eta_{k}^{2} \eta_{i}^{2}+\frac{\partial a_{1}}{\partial w}(x+2 \mu u) \\
& +\sum_{i=1}^{4} \frac{\partial a_{1}}{\partial \eta^{i}} \eta_{i}^{2}+a_{2}\left(w, \eta^{i}\right) \\
& c_{3}=\frac{\partial^{2} a_{0}}{\partial w^{2}}(x+2 \mu u) u \\
& +\sum_{i=1}^{4} \frac{\partial^{2} a_{0}}{\partial w \partial \eta^{i}} u \eta_{i}^{2}+\frac{1}{6} \frac{\partial^{3} a_{0}}{\partial w^{3}}(x+2 \mu u)^{3} \\
& +\frac{1}{2} \sum_{i=1}^{4} \frac{\partial^{3} a_{0}}{\partial w^{2} \partial \eta^{i}}(x+2 \mu u)^{2} \eta_{i}^{2} \\
& +\frac{1}{2} \sum_{i, k=1}^{4} \frac{\partial^{3} a_{0}}{\partial w \partial \eta^{i} \partial \eta^{k}}(x+2 \mu u) \eta_{k}^{2} \eta_{i}^{2} \\
& +\frac{1}{6} \sum_{i, k, l=1}^{4} \frac{\partial^{3} a_{0}}{\partial \eta^{i} \partial \eta^{k} \partial \eta^{l}} \eta_{l}^{2} \eta_{k}^{2} \eta_{i}^{2} \\
& +\frac{\partial a_{1}}{\partial w} u+\frac{1}{2} \frac{\partial^{2} a_{1}}{\partial w^{2}}(x+2 \mu u)^{2} \\
& +\sum_{i=1}^{4} \frac{\partial^{2} a_{1}}{\partial w \partial \eta^{i}}(x+2 \mu u) \eta_{i}^{2} \\
& +\frac{1}{2} \sum_{i, k=1}^{4} \frac{\partial^{2} a_{1}}{\partial \eta^{i} \partial \eta^{k}} \eta_{k}^{2} \eta_{i}^{2}+\frac{\partial a_{2}}{\partial w}(x+2 \mu u) \\
& +\sum_{i=1}^{4} \frac{\partial a_{2}}{\partial \eta^{i}} \eta_{i}^{2}+a_{3}\left(w, \eta^{i}\right)
\end{aligned}
$$

From the expressions, we know that $c_{j}$ 's are $n \times 1$ matrix functions of $\left(w, \eta^{i}\right)$ and depend on $w$ rationally.

For $k \geq 1$, let $T_{k, \epsilon}=F_{k-1, \epsilon}, P_{k, \epsilon}=T_{k, \epsilon}\left(T_{k, \epsilon}^{\dagger} T_{k, \epsilon}\right)^{-1} T_{k, \epsilon}^{\dagger}$. Define $\psi_{k, \epsilon}$ and $\widehat{\psi}_{k}$ by induction as follows:

$$
\begin{aligned}
& \psi_{1, \epsilon}=\psi_{\mu+\epsilon, P_{1, \epsilon},} \quad \widehat{\psi}_{1}=\lim _{\epsilon \rightarrow 0} \psi_{1, \epsilon}, \\
& \psi_{k, \epsilon}=\psi_{\mu+\epsilon, P_{k, \epsilon}} \sharp \widehat{\psi}_{k-1}, \quad \widehat{\psi}_{k}=\lim _{\epsilon \rightarrow 0} \psi_{k, \epsilon} \text {. }
\end{aligned}
$$

Let $\widetilde{T}_{k, \epsilon}=\widehat{\psi}_{k-1}(\mu+\epsilon) T_{k, \epsilon}, \widetilde{P}_{k, \epsilon}=\widetilde{T}_{k, \epsilon}\left(\widetilde{T}_{k, \epsilon}^{\dagger} \widetilde{T}_{k, \epsilon}\right)^{-1} \widetilde{T}_{k, \epsilon}^{\dagger}$; then $\psi_{k, \epsilon}=\psi_{\mu+\epsilon, \widetilde{P}_{k, \epsilon}} \widehat{\psi}_{k-1}$, where implicit “ $\star$ ” products are still assumed between classical fields and their components.

Theorem 7. Let $a_{0}, a_{1}, \ldots$ be a sequence of $n \times 1$ matrix functions of $w$ and $\eta^{i}$ and depend on $w$ rationally. Let $T_{k, \epsilon}, P_{k, \epsilon}$, $\psi_{k, \epsilon}, \widehat{\psi}_{k}$, and $\widetilde{T}_{k, \epsilon}$ be defined as above. Then one has

(1) $\widetilde{T}_{k, \epsilon}=\widehat{T}_{k}+\epsilon S_{k, 1}+\epsilon^{2} S_{k, 2}+\cdots$, where $\widehat{T}_{k}=c_{0}+$ $\sum_{j=1}^{k-1}(\mu-\bar{\mu})^{j} P_{k-1, j} c_{j}, P_{l, j}=\sum_{l \geq i_{1}>\cdots>i_{j} \geq 1} \widehat{P}_{i_{1}}^{\perp} \cdots \widehat{P}_{i_{j}}^{\perp}$, and $\widehat{P}_{i}=\widehat{T}_{i}\left(\widehat{T}_{i}^{\dagger} \widehat{T}_{i}\right)^{-1} \widehat{T}_{i}^{\dagger}$;
(2) $\widehat{\psi}_{k}=\psi_{\mu, \widehat{P}_{k}} \cdots \psi_{\mu, \widehat{P}_{1}}$ is a multisoliton solution of (4) with only a pole at $\zeta=\mu$ of order $k$;

(3) the associated superfield is $\widehat{\Phi}_{k}=\left(\widehat{P}_{1}+\right.$ $\left.(\mu / \bar{\mu}) \widehat{P}_{1}^{\perp}\right) \cdots\left(\widehat{P}_{k}+(\mu / \bar{\mu}) \widehat{P}_{k}^{\perp}\right)$.

Proof. We prove the theorem by induction on $k$. For $k=1$, the theorem is clearly true. Suppose the theorem is true for $k$, we will prove that (1)-(3) hold for $k+1$.

(1) By Theorem 3 and induction hypothesis,

$$
\begin{aligned}
& \widetilde{T}_{k+1, \epsilon}=\widehat{\psi}_{k}(\mu+\epsilon) T_{k+1, \epsilon} \\
& =\left(I_{n}+\frac{\mu-\bar{\mu}}{\epsilon} \widehat{P}_{k}^{\perp}\right) \widehat{\psi}_{k-1}(\mu+\epsilon) F_{k, \epsilon} \\
& =\left(I_{n}+\frac{\mu-\bar{\mu}}{\epsilon} \widehat{P}_{k}^{\perp}\right) \widehat{\psi}_{k-1}(\mu+\epsilon) \\
& \times\left(F_{k-1, \epsilon}+a_{k}\left(w_{\epsilon}, \eta_{\epsilon}^{i}\right) \epsilon^{k}\right) \\
& =\left(I_{n}+\frac{\mu-\bar{\mu}}{\epsilon} \widehat{P}_{k}^{\perp}\right) \\
& \times\left(\widehat{\psi}_{k-1}(\mu+\epsilon) T_{k, \epsilon}+\widehat{\psi}_{k-1}(\mu+\epsilon) a_{k}\left(w_{\epsilon}, \eta_{\epsilon}^{i}\right) \epsilon^{k}\right) \\
& =\left(I_{n}+\frac{\mu-\bar{\mu}}{\epsilon} \widehat{P}_{k}^{\perp}\right)\left(\widetilde{T}_{k, \epsilon}+\left(I_{n}+\frac{\mu-\bar{\mu}}{\epsilon} \widehat{P}_{k-1}^{\perp}\right)\right. \\
& \cdots\left(I_{n}+\frac{\mu-\bar{\mu}}{\epsilon} \widehat{P}_{1}^{\perp}\right) \\
& \left.\times a_{k}\left(w_{\epsilon}, \eta_{\epsilon}^{i}\right) \epsilon^{k}\right) \\
& =\left(I_{n}+\frac{\mu-\bar{\mu}}{\epsilon} \widehat{P}_{k}^{\perp}\right)\left(\widehat{T}_{k}+\epsilon S_{k, 1}+\epsilon(\mu-\bar{\mu})^{k-1}\right. \\
& \left.\times P_{k-1, k-1} a_{k}\left(w, \eta^{i}\right)+O\left(\epsilon^{2}\right)\right) \\
& =\widehat{T}_{k}+(\mu-\bar{\mu}) \widehat{P}_{k}^{\perp}\left(S_{k, 1}+(\mu-\bar{\mu})^{k-1}\right. \\
& \left.\times P_{k-1, k-1} a_{k}\left(w, \eta^{i}\right)\right)+O(\epsilon) .
\end{aligned}
$$

In the last step we have used $\widehat{P}_{k}^{\perp} \widehat{T}_{k}=0$. Thus all terms of negative powers of $\epsilon$ vanish in the Laurent series expansion of $\widetilde{T}_{k+1, \epsilon}$ in $\epsilon$; hence

$$
\begin{aligned}
\widetilde{T}_{k+1, \epsilon}= & \widehat{\psi}_{k}(\mu+\epsilon) F_{k, \epsilon} \\
= & \left(I_{n}+\frac{\mu-\bar{\mu}}{\epsilon} \widehat{P}_{k}^{\perp}\right) \cdots\left(I_{n}+\frac{\mu-\bar{\mu}}{\epsilon} \widehat{P}_{1}^{\perp}\right) \\
& \times\left(c_{0}+c_{1} \epsilon+\cdots+c_{k} \epsilon^{k}+O\left(\epsilon^{k+1}\right)\right) \\
= & \left(I_{n}+\frac{\mu-\bar{\mu}}{\epsilon} P_{k, 1}+\cdots+\frac{(\mu-\bar{\mu})^{k}}{\epsilon^{k}} P_{k, k}\right) \\
& \times\left(c_{0}+c_{1} \epsilon+\cdots+c_{k} \epsilon^{k}+O\left(\epsilon^{k+1}\right)\right)
\end{aligned}
$$




$$
\begin{aligned}
= & c_{0}+(\mu-\bar{\mu}) P_{k, 1} c_{1} \\
& +\cdots+(\mu-\bar{\mu})^{k} P_{k, k} c_{k}+O(\epsilon) .
\end{aligned}
$$

Therefore we obtain

$$
\widehat{T}_{k+1}=\lim _{\epsilon \rightarrow 0} \widetilde{T}_{k+1, \epsilon}=c_{0}+\sum_{j=1}^{k}(\mu-\bar{\mu})^{j} P_{k, j} c_{j} .
$$

(2) By the inductive definition before this theorem,

$$
\begin{aligned}
\widehat{\psi}_{k+1} & =\lim _{\epsilon \rightarrow 0} \psi_{\mu+\epsilon, P_{k+1, \epsilon}} \sharp \widehat{\psi}_{k}=\lim _{\epsilon \rightarrow 0} \psi_{\mu+\epsilon, \widetilde{P}_{k+1, \epsilon}} \widehat{\psi}_{k} \\
& =\psi_{\mu, \widehat{P}_{k+1}} \widehat{\psi}_{k}=\psi_{\mu, \widehat{P}_{k+1}} \psi_{\mu, \widehat{P}_{k}} \cdots \psi_{\mu, \widehat{P}_{1}} \\
& =\left(I_{n}+\frac{\mu-\bar{\mu}}{\zeta-\mu} \widehat{P}_{k+1}^{\perp}\right) \cdots\left(I_{n}+\frac{\mu-\bar{\mu}}{\zeta-\mu} \widehat{P}_{1}^{\perp}\right) \\
& =I_{n}+\sum_{j=1}^{k+1} \frac{(\mu-\bar{\mu})^{j}}{(\zeta-\mu)^{j}} P_{k+1, j} .
\end{aligned}
$$

By Theorem 3, $\psi_{\mu+\epsilon, P_{k+1 \epsilon}} \sharp \widehat{\psi}_{k}$ is a solution of (4) for small $|\epsilon|>$ 0 . By continuity, so is $\widehat{\psi}_{k+1}$. The coefficient of $(\zeta-\mu)^{-k-1}$ of $\widehat{\psi}_{k+1}$ is $(\mu-\bar{\mu})^{k+1} P_{k+1, k+1}$. To show that $\widehat{\psi}_{k+1}$ has a pole at $\zeta=\mu$ of multiplicity $k+1$, it suffices to show that

$$
P_{k+1, k+1}=\widehat{P}_{k+1}^{\perp} \widehat{P}_{k}^{\perp} \cdots \widehat{P}_{1}^{\perp} \neq 0 .
$$

As in the nonsupersymmetric scenario, we choose the operator formalism to prove (44). So now the Hermitian projections $\widehat{P}_{i}, i=1, \ldots, k+1$,

$$
\begin{aligned}
& P_{k+1, k+1}=\widehat{P}_{k+1}^{\perp} \widehat{P}_{k}^{\perp} \cdots \widehat{P}_{1}^{\perp}, \\
& P_{l, j}=\sum_{l \geq i_{1}>\cdots>i_{j} \geq 1} \widehat{P}_{i_{1}}^{\perp} \cdots \widehat{P}_{i_{j}}^{\perp}
\end{aligned}
$$

(under the Moyal-Weyl map) are $U(n)$-valued matrices, and the products in (45) are the ordinary products instead of star products. We write $P_{k, j}=P_{k-1, j}+\widehat{P}_{k}^{\perp} P_{k-1, j-1}$. So (42) for $k+1$ can be written as

$$
\begin{aligned}
\widehat{T}_{k+1}= & c_{0}+\sum_{j=1}^{k-1}(\mu-\bar{\mu})^{j} P_{k-1, j} c_{j} \\
& +\widehat{P}_{k}^{\perp} \sum_{j=0}^{k-1}(\mu-\bar{\mu})^{j+1} P_{k-1, j} c_{j+1} \\
= & \widehat{T}_{k}+(\mu-\bar{\mu}) \widehat{P}_{k}^{\perp} \sum_{j=0}^{k-1}(\mu-\bar{\mu})^{j} P_{k-1, j} c_{j+1} .
\end{aligned}
$$

By the induction hypothesis, $\widehat{T}_{k} \neq 0$, then the above formula implies that

$$
\operatorname{Im} \widehat{P}_{k+1} \cap \operatorname{Im} \widehat{P}_{k}^{\perp}=0 .
$$

If (44) does not hold, that is, $\widehat{P}_{k+1}^{\perp} \widehat{P}_{k}^{\perp} \cdots \widehat{P}_{1}^{\perp}=0$, then $\widehat{P}_{k}^{\perp} \cdots \widehat{P}_{1}^{\perp} \subset \operatorname{Im} \widehat{P}_{k+1}$, but $\widehat{P}_{k}^{\perp} \cdots \widehat{P}_{1}^{\perp} \neq 0$ by induction hypothesis, and clearly $\widehat{P}_{k}^{\perp} \cdots \widehat{P}_{1}^{\perp} \subset \operatorname{Im} \widehat{P}_{k}^{\perp}$, so we have

$$
0 \neq \widehat{P}_{k}^{\perp} \cdots \widehat{P}_{1}^{\perp} \subset \operatorname{Im} \widehat{P}_{k+1} \cap \operatorname{Im} \widehat{P}_{k}^{\perp},
$$

which contradicts (47). Therefore (2) holds for $k+1$.

(3) The expression for the associated superfield $\widehat{\Phi}_{k+1}$ is straightforward; that is

$$
\begin{aligned}
\widehat{\Phi}_{k+1} & =\widehat{\psi}_{k+1}^{-1}(\zeta=0) \\
& =\left(\widehat{P}_{1}+\frac{\mu}{\bar{\mu}} \widehat{P}_{1}^{\perp}\right) \cdots\left(\widehat{P}_{k}+\frac{\mu}{\bar{\mu}} \widehat{P}_{k}^{\perp}\right)\left(\widehat{P}_{k+1}+\frac{\mu}{\bar{\mu}} \widehat{P}_{k+1}^{\perp}\right) .
\end{aligned}
$$

The proof is completed.

Note that all $\widehat{P}_{j}$ 's are of rank one in the above construction. But the same limiting method also produces multisoliton solutions of the form $\psi_{\mu, \widehat{P}_{k}} \cdots \psi_{\mu, \widehat{P}_{1}}$ with $\operatorname{rank}\left(\widehat{P}_{1}\right) \geq \cdots \geq$ $\operatorname{rank}\left(\widehat{P}_{k}\right)$. Similar computations give the construction of multisoliton solutions with pole data $(\mu, k)$ and arbitrary rank data $\left(n_{1}, \ldots, n_{k}\right)$.

Like the bosonic case, we can also associate to each multisoliton solution of (4) with pole data $(\mu, k)$ a generalized algebraic BT. Using these generalized BTs, we can construct multisoliton solutions with general pole data $\left(\mu_{1}, \ldots, \mu_{k}, n_{1}, \ldots, n_{k}\right)$.

\section{Conflict of Interests}

The authors declare that there is no conflict of interests regarding the publication of this paper.

\section{Acknowledgment}

This work was supported by the Key Project of Jiangsu Second Normal University (JSNU-Z-4469).

\section{References}

[1] R. S. Ward, "Soliton solutions in an integrable chiral model in $2+1$ dimensions," Journal of Mathematical Physics, vol. 29, no. 2, pp. 386-389, 1988.

[2] B. Dai and C.-L. Terng, "Bäcklund transformations, Ward solitons, and unitons," Journal of Differential Geometry, vol. 75, no. 1, pp. 57-108, 2007.

[3] O. Lechtenfeld and A. D. Popov, "Noncommutative solitons in a supersymmetric chiral model in $2+1$ dimensions," Journal of High Energy Physics, no. 6, article 065, pp. 1-28, 2007.

[4] T. Ioannidou, "Soliton solutions and nontrivial scattering in an integrable chiral model in $(2+1)$ dimensions," Journal of Mathematical Physics, vol. 37, no. 7, pp. 3422-3441, 1996.

[5] R. S. Ward, "Nontrivial scattering of localized solitons in a $(2+$ 1)-dimensional integrable system," Physics Letters A, vol. 208, no. 3, pp. 203-208, 1995. 
[6] O. Lechtenfeld and A. D. Popov, "Scattering of noncommutative solitons in $2+1$ dimensions," Physics Letters B, vol. 523, no. 1-2, pp. 178-184, 2001.

[7] O. Lechtenfeld and A. D. Popov, "Non-commutative multisolitons in $2+1$ dimensions," Journal of High Energy Physics, no. 11, article 40, pp. 1-32, 2001.

[8] C. Gutschwager, O. Lechtenfeld, and T. A. Ivanova, "Scattering of noncommutative waves and solitons in a supersymmetric chiral model in 2+1 dimensions," Journal of High Energy Physics, no. 11, article 052, pp. 1-16, 2007.

[9] X.-j. Zhu, "Noncommutative multi-solitons in a modified chiral model in $2+1$ dimensions," Communications in Mathematical Research, vol. 25, no. 4, pp. 349-360, 2009.

[10] O. Lechtenfeld, A. D. Popov, and B. Spendig, "Noncommutative solitons in open $N=2$ string theory," Journal of High Energy Physics, no. 6, article 11, pp. 1-18, 2001.

[11] K. Efetov, Supersymmetry in Disorder and Chaos, Cambridge University Press, Cambridge, UK, 1997. 


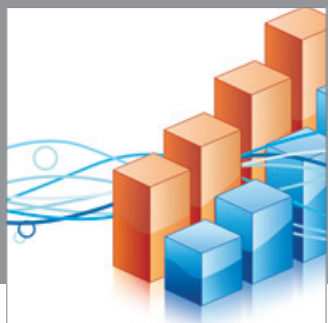

Advances in

Operations Research

mansans

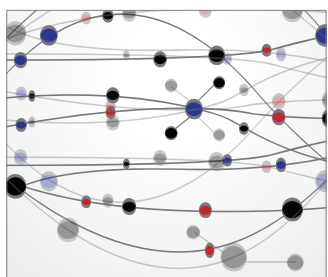

The Scientific World Journal
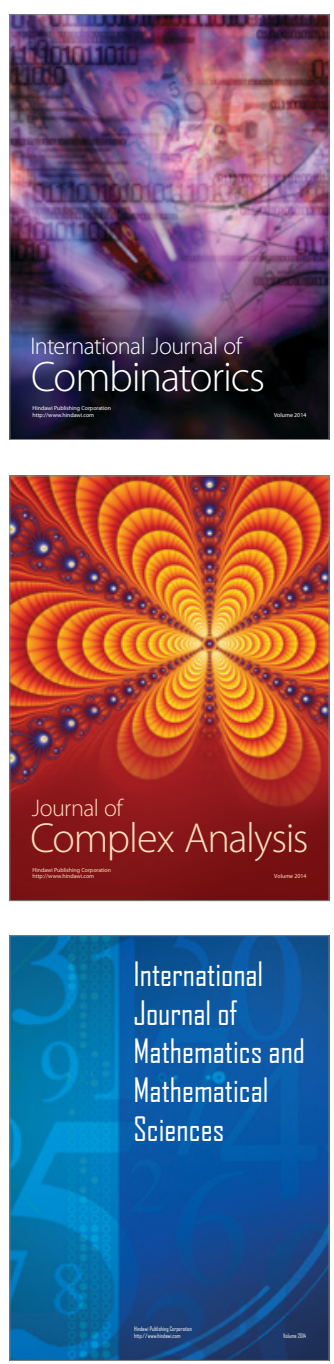
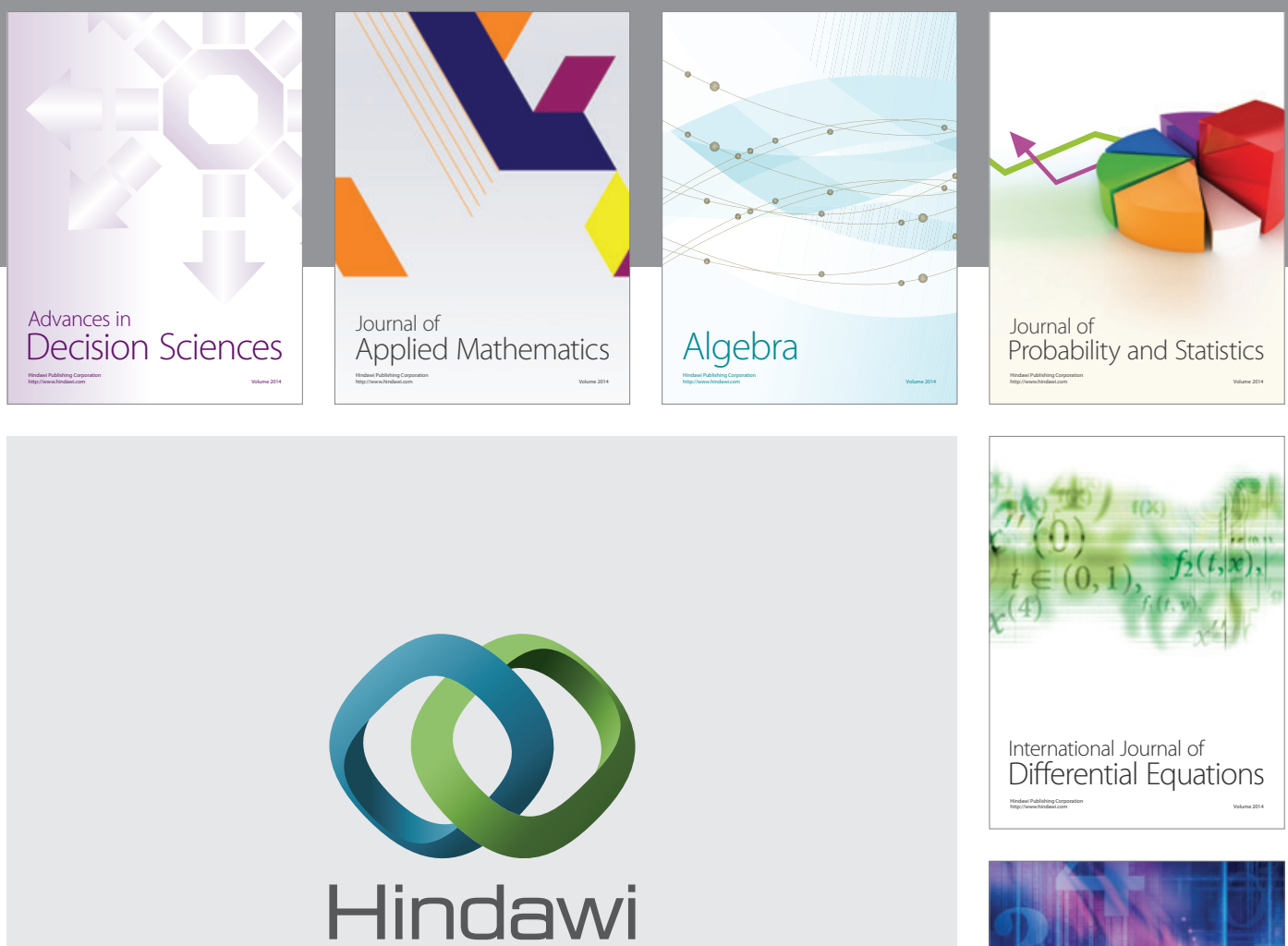

Submit your manuscripts at http://www.hindawi.com
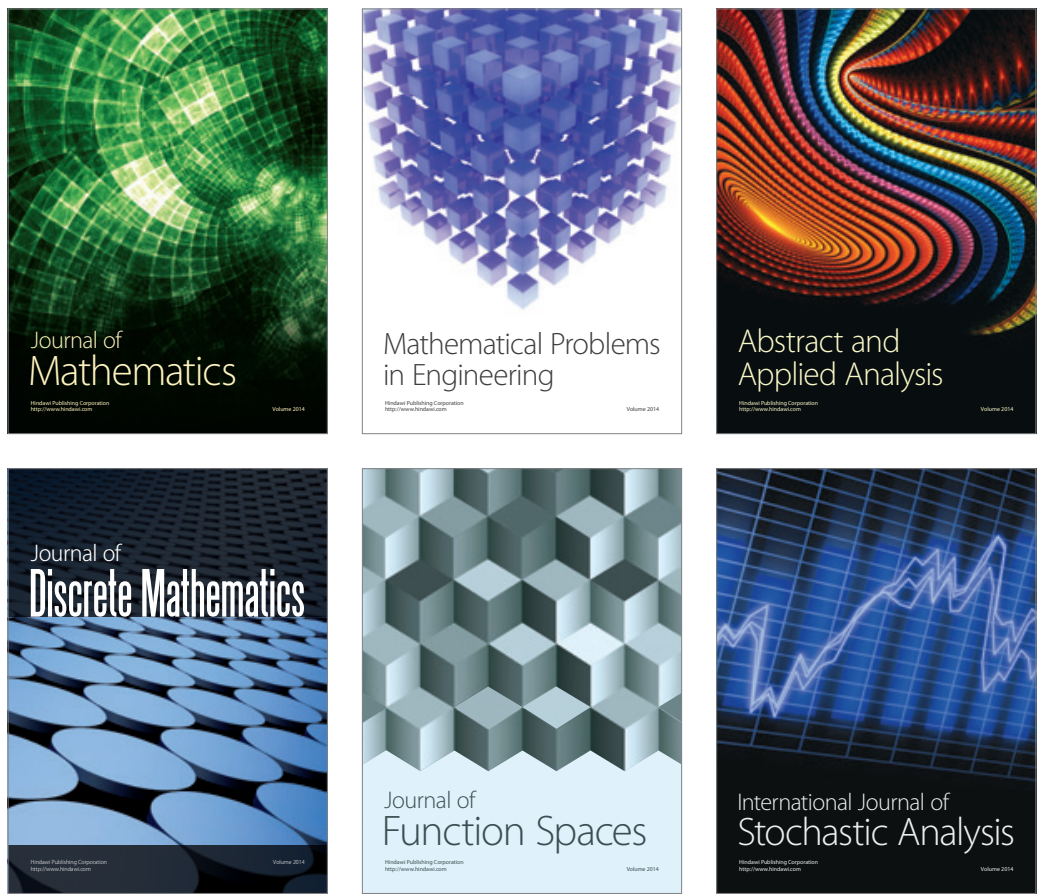

Journal of

Function Spaces

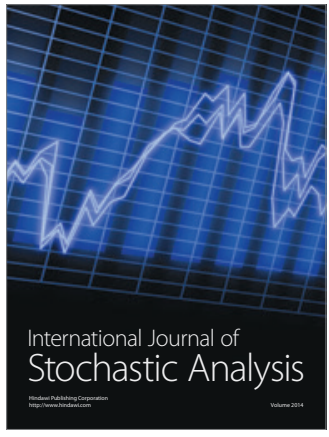

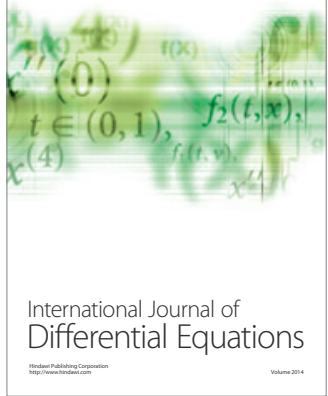
